\title{
La libertad de los periodistas en Chile y el mundo: los niveles y orígenes de las restricciones percibidas por los periodistas en su trabajo ${ }^{1}$
}

\author{
Journalistic freedom in Chile and the world: levels and origins of \\ restrictions as perceived by journalists in their work
}

\author{
Ewa Sapiezynska \\ Universidad de Chile \\ eva.sap@gmail.com
}

\begin{abstract}
Resumen
Usando la base de datos de Worlds of Journalism, este artículo presenta índices de restricciones percibidas por los periodistas en su trabajo diario. Uno de los principales resultados es que los niveles de restricción son considerablemente más altos en Chile que en el mundo. Las diferencias crecen aún más si comparamos Chile solamente con otros países democráticos, sobre todo en la dimensión de restricción por el mercado y la publicidad, donde un $67 \%$ de los periodistas chilenos se ubica en los niveles altos de restricción, superando casi 3 veces el resultado de los países democráticos en general. Los niveles de restricción son también muy altos en Chile en la dimensión de restricción por parte de los superiores y dueños de los medios, superando nuevamente los niveles mundiales.
\end{abstract}

Palabras Clave: Periodismo, libertad de expresión, libertad de prensa, derecho a la información, índices de restricción.

\begin{abstract}
Using the Worlds of Journalism database, this article presents indices of restriction as perceived by journalists in their daily work. One of the main findings is that the level of restriction found in Chile is considerably higher than the world average. This difference is even starker if Chile is compared only with other democratic countries. This is especially the case in terms of restrictions imposed by the market
\end{abstract}

\footnotetext{
1 Agradezco el apoyo económico del Programa MECE Educación Superior (2) del Doctorado en Ciencias Sociales de la Universidad de Chile. Agradezco además los comentarios del Dr. Jaime Fierro a la versión preliminar de este artículo.
} 
and advertising, a dimension where $67 \%$ of Chilean journalists perceive high levels of restriction, surpassing almost 3 times the result for democratic countries in general. The restriction levels are also very high in Chile in the dimension of restriction by superiors and media owners, again surpassing world levels.

Keywords: Journalism, freedom of speech, press freedom, information right, restriction indexes.

Los medios de comunicación son una de las principales arenas para el ejercicio de la libertad de expresión y el derecho a la información de todos los ciudadanos, jugando un rol central en el debate público y en la formación de voluntad política (Cottle, 2003; Murdock, 1999; Schudson, 2002). De allíla importancia de diagnosticar el estado del periodismo e investigar de dónde vienen y qué peso relativo tienen las restricciones de la libertad de expresión que perciben los periodistas en su trabajo.

Según los estudios existentes, tan solo uno de cada tres chilenos percibe la libertad de expresión en el país como completamente garantizada (Latinobarómetro, 2009). Los chilenos opinan que los medios, y especialmente los diarios impresos, tienen poca independencia frente a poderes como el gobierno (29\%), avisadores (28\%), iglesia católica (26\%) y grupos empresariales (25\%) (UDP, 2010). Además, en 2012 la ya baja confianza en los medios en Chile cayó drásticamente, reduciéndose el porcentaje de los que confían en la televisión en más de 17 puntos porcentuales, de un $36 \%$ al 18,5\% (UDP, 2012). A la vez, Chile es un ejemplo límite de la concentración económica de los medios, que, aunque constituye un fenómeno global, ha alcanzado en el país niveles extremadamente altos, sobre todo en el mercado de la prensa, donde tan sólo dos empresas -COPESA y El Mercurio- controlan aproximadamente un 95\% de los diarios en el país (Gumucio y Parrini, 2009). Es preocupante también la creciente concentración de la radiofonía en Chile, donde el Grupo Prisa tiene 12 señales de radio que captan un 37,4\% de la inversión publicitaria destinada al sector y reúnen un 44,7\% de audiencia en Santiago y 38,9\% en las regiones (AMARC, 2009). La desaparición de los medios públicos, la total dependencia de los medios de los ingresos de la publicidad y la falta de políticas públicas referidas a la comunicación son otros aspectos alarmantes. Se suma a este escenario la situación socio-laboral de los periodistas: el mercado laboral está saturado con aproximadamente 1200 periodistas que se titulan cada año, para competir por unos 500 puestos de trabajo. Solo un tercio de los periodistas chilenos está colegiado, algunos son despedidos por querer organizarse y el ingreso mensual de muchos de ellos no supera los $500 \mathrm{mil}$ pesos $^{2}$.

2 Datos del Colegio de Periodistas de Chile. Entrevista al Presidente del Colegio, Marcelo Castillo, $4 / 12 / 2012$. 
A pesar de ello, Chile suele obtener muy buenos puntajes en los índices de libertad de expresión y prensa de las organizaciones internacionales Reporteros Sin Fronteras y Freedom House, siendo excepción la evaluación correspondiente al año 2011, cuando en las grandes marchas estudiantiles fueron atacados y arrestados varios periodistas (Sapiezynska, Lagos, y Cabalín, 2013). Estas mediciones predominan en el debate sobre el tema, mientras que escasean índices académicos de libertad de expresión y prensa. Del mismo modo, no existe un acuerdo en la literatura sobre las dimensiones de las restricciones que viven los periodistas ni su eventual jerarquía (Hanitzsch et al., 2010). Hacer una modesta propuesta en este campo será el primer paso de esta investigación. Luego, usando datos recogidos por el proyecto Worlds of Journalism en 21 países, se construirá una serie de índices de los niveles de restricción de la libertad de los periodistas dentro de una lógica comparativa entre Chile y el mundo.

Las preguntas de investigación apuntan a los siguientes aspectos: ¿Cuáles son los niveles de restricción que perciben los periodistas en su trabajo? ¿De dónde provienen dichas restricciones?, es decir, ¿cuáles son las dimensiones de las restricciones? ¿En cuál de las dimensiones las restricciones llegan a niveles más altos? ¿Hay diferencias sustanciales entre estos niveles en Chile y en el mundo? ¿Hay diferencias entre estos niveles en Chile y en otros países democráticos?

El objetivo general de este estudio es analizar y comparar los niveles de restricción que encuentran los periodistas en su trabajo diario en el mundo y en Chile. Los objetivos específicos son:

- Construir subíndices (o dimensiones) de diferentes tipos de restricciones y un índice general de niveles de restricción percibidos por los periodistas.

- Crear conocimiento relevante para el debate académico y político sobre las restricciones a la libertad de expresión en el ámbito mediático en Chile.

\section{RESTRICCIONES A LA LIBERTAD PERIODÍSTICA}

El tema de las limitaciones de la libertad de los periodistas constituye un pequeño campo en la investigación sobre los medios de comunicación, aunque hay que señalar que los estudios al respecto, más aún los comparados, se han hecho casi exclusivamente en Europa y Estados Unidos 3 . Sistematizando estas investigaciones, Hanitzsch y Mellado (2011), distinguen el enfoque en el ámbito político como determinante para las restricciones (Blumer y Gurevitch, 1990). En otra línea, se ha investigado el ámbito económico (Murdock, 2001) y en la tercera, el ámbito organizacional

3 Siendo por ello novedoso el estudio global de Worlds of Journalism, cuya base de datos de acceso abierto fue empleada en el estudio. 
relacionado con las prácticas y rutinas profesionales de los periodistas y las relaciones de poder dentro de los medios (Tuchman, 1978). Hay también investigaciones que se centran en el nivel individual del periodista: cómo su posición política, gustos y el entendimiento de su rol dan forma al trabajo informativo y restringen la llegada de ciertos tipos de noticias al público (Breed, 1955). No obstante, algunos de los autores mencionados incursionan en varios ámbitos a la vez, interesándose Blumer y Gurevitch además por restricciones económicas, institucionales y las provenientes de las audiencias; subrayando Murdock que las restricciones económicas muchas veces van acompañadas de las políticas, y fundando Breed las bases para lo que se desarrolló más tarde como investigación organizacional.

Hay también en la literatura intentos de modelar estas dimensiones o niveles de restricción e influencia, siendo el más conocido el modelo de Shoemaker y Reese (1996), el cual plantea una jerarquía de cinco niveles de influencia sobre los contenidos mediáticos: el nivel individual del periodista, el nivel de las prácticas del oficio, el nivel organizacional que apunta a las dinámicas y relaciones de poder dentro del medio, el nivel extra-mediático llamado institucional y, el más alto, llamado ideológico o sociocultural. En tanto los modelos anteriores hablaban de 3 niveles -individual, organizacional e institucional-, los modelos actuales incluyen varios otros ámbitos (Hanitzsch y Mellado, 2011). En el presente trabajo, las dimensiones de la restricción emergerán de un análisis factorial, es decir, el primer criterio será estadístico y las consideraciones teóricas ayudarán en la posterior eliminación de las dimensiones y variables con menos relevancia.

La construcción de índices que determinan los niveles de libertad y restricción, tal como se señaló, es un campo en gran parte abandonado por los académicos a las organizaciones internacionales defensoras de la libertad de expresión, cuyas metodologías parecen captar mucho mejor las restricciones a la libertad de expresión presentes en las dictaduras, que los sutiles mecanismos de las democracias. De allí que sea necesario buscar instrumentos nuevos, adaptados a detectar las restricciones propias de las democracias formales. Uno de los aspectos dignos de un análisis más detallado al respecto son las presiones comerciales y organizacionales. Según Hanitzsch y Mellado (2011), los periodistas chilenos perciben el ámbito económico, junto con el ámbito organizacional, como las dos dimensiones con más influencia sobre su trabajo diario. A la vez, Chile es el país donde las influencias económicas sobre el periodismo son percibidas como las más fuertes entre los 18 países que

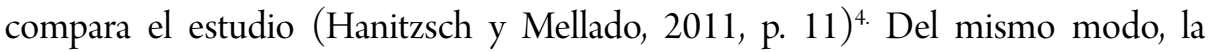
publicación de Sapiezynska, Lagos y Cabalín (2013), apoyada en otra base de datos, apunta a que los niveles de restricción percibidos por los periodistas en Chile son

4 Nuevamente, lo medido fueron las percepciones de los mismos periodistas. 
altos, particularmente en el subíndice de restricción por parte de los superiores en el lugar de trabajo.

Las hipótesis de la presente investigación toman como punto de partida las conclusiones de Hanitzsch y Mellado (2011) y las de Sapiezynska, Lagos y Cabalín (2013), pero van más allá de ellas:

- H1: Se espera que entre un 40 y un 50\% de los periodistas a nivel global reporte niveles regulares de restricción según el índice general ${ }^{5}$ y no más de un tercio reporte niveles altos en el mundo. En Chile se espera que los niveles del índice general sean altos para más de la mitad de los periodistas.

- H2: En el mundo los niveles más altos de restricción se encontrarán en el índice de restricciones por agentes internos (superiores y dueños del medio), llegando al nivel alto para más de la mitad de los periodistas. En Chile este índice tendrá valores todavía más altos.

- H3: Los niveles más altos en Chile se encontrarán en el índice de las restricciones por el mercado y la publicidad.

- H4: Los niveles de restricción serán considerablemente más altos en Chile que en los países democráticos en general, sobre todo en la dimensión referida al mercado, donde se espera que el porcentaje de los periodistas que percibe niveles altos de restricción en Chile supere por lo menos dos veces este porcentaje en los países democráticos.

\section{ASPECTOS METODOLÓGICOS}

El presente artículo usa la base de datos del estudio piloto de un grupo internacional de investigadores de las comunicaciones llamado Worlds of Journalism. Este estudio se realizó entre 2007 y 2011 y su base de datos fue hecha pública en 2012. En su elaboración participaron, entre otros, los académicos ya citados: Hanitzsch y Mellado, la última siendo responsable de los datos correspondientes a Chile. El universo fueron los periodistas de los medios locales y nacionales (prensa, radio, tv y agencias de prensa) de 21 países: Alemania, Australia, Austria, Brasil, Bulgaria, Chile, China, Egipto, España, Estados Unidos, Grecia, Indonesia, Israel, México, Paquistán, Portugal, Romania, Rusia, Suiza, Turquía y Uganda. El muestreo se hizo de una manera análoga en cada uno de los países usando un esquema de cuota establecido.

5 Véase más adelante sobre su construcción. 
En cada país se eligieron 20 medios (según criterios preestablecidos que se referían al soporte y carácter del medio y su representatividad ${ }^{6}$ ) y en cada uno de estos medios se eligieron 5 periodistas de diferentes cargos en la jerarquía interna, de allí que la muestra sean 100 periodistas encuestados en cada país, 2100 casos en total'.

Para el presente trabajo fueron recodificadas de menos a más todas las variables que se incluyeron posteriormente en el análisis factorial y pasaron a formar parte de subíndices (dimensiones), sin que cambiara la escala original 1-5. Además, los valores no aplica (8), no sabe (77) y no responde (99) fueron recodificados al valor 100 , lo que posteriormente ayudó a crear un mecanismo de no eliminar como casos perdidos de las dimensiones a encuestados que no respondieron una vez (es decir, los valores perdidos fueron desde 200 mientras que los valores 101 pasaron a ser 1, 102 pasaron a ser 2 , etc.).

Se creó una variable nueva, nominal tipo dummy, que se refiere a que si el país es democrático (valor 1) o enfrenta faltas severas a la democracia (valor 0). Para ello fue recodificada la variable original Countryl. La división de los países en los democráticos y poco democráticos se basó en informes de Freedom House, Idea y Human Rights Watch ${ }^{8}$. En efecto, se establecieron como países democráticos: Alemania, Australia, Austria, Brasil, Bulgaria, Chile, España, Estados Unidos, Grecia, Israel, México, Portugal, Romania, Suiza y Turquía. Y como países con faltas severas a la democracia: China, Egipto, Indonesia, Paquistán, Rusia y Uganda. La creación de esta variable posibilita comparar Chile con el mundo en general, representado por los 21 países que incluye la base de datos, y además permite compararlo con las otras democracias formales cuyos modelos y propuestas legales suelen considerarse en Chile a la hora de elaborar políticas públicas.

\section{CONSTRUCCIÓN DE ÍNDICES}

Primero, mediante el análisis factorial, se decidió cuáles variables serían las que compondrían cada dimensión?. Posteriormente, para calcular el valor de cada

\footnotetext{
63 diarios nacionales (2 enfocando en asuntos ciudadanos y uno con enfoque más consumista), 4 locales (3 ciudadanos, 1 consumista), 2 revistas (una más ciudadana otra consumista), 1 agencia de prensa, 4 estaciones tv nacionales ( 3 privadas y 1 estatal o pública), 2 estaciones de tv locales ( 1 privada, 1 estatal o pública), 2 estaciones de radio nacionales (1 privada, la otra estatal o pública) y 2 estaciones de radio locales con la misma división acerca de la propiedad.

7 Las encuestas fueron cara a cara o por teléfono. Adicionalmente, se buscó información sobre cada uno de los medios donde trabajaban los entrevistados y sobre los sistemas mediáticos en todos los 21 países.

8 www.freedomhouse.org, www.idea.int y www.hrw.org

9 Véase análisis factorial más adelante.
} 
dimensión se sumaron sus componentes y la suma fue dividida por el número de componentes, para que el valor de la dimensión correspondiera a la misma escala de 1 a 5 , según la cual fueron medidos sus componentes. Debido a la mencionada recodificación de valores entre 100 y 200, cuyo objetivo fue reducir el porcentaje de casos perdidos y la posterior división del valor de la dimensión por el número de componentes, los valores de las dimensiones no son necesariamente números naturales y pueden tener decimales ${ }^{10}$.

El índice general es, analógicamente, una suma de las 4 dimensiones que lo componen y sus valores corresponden a la suma de los valores de las dimensiones dividida por 4. De allí que la construcción del índice general pueda presentarse como la siguiente ecuación:

\section{Índice general $=$ (dimensión “agentes externos" + dimensión "mercado y publicidad" + dimensión "leyes y convenciones" + dimensión “agentes internos”) / 4}

$\mathrm{Al}$ igual que las dimensiones, el índice general así calculado es intervalar con valores de 0 a 5 , pasando por valores con decimales. Luego, para las distribuciones de frecuencias, se recodificaron el índice y las dimensiones pasando a ser variables ordinales con tan solo 3 valores (1, 2 y 3 ): los valores previos $0-2$ pasaron a ser 1 , que significa "no hay restricción o es de bajo nivel"; los valores 2,0001-3 corresponden ahora a 2, es decir, "nivel regular"; mientras que valores previamente mayores a 3 corresponden al nuevo valor 3: "nivel alto".

\section{ANÁLISIS FACTORIAL}

La base de datos contenía 29 variables referidas a las limitaciones que perciben los periodistas sobre su trabajo. Se usó el tipo de análisis factorial llamado Análisis de Componentes Principales (ACP) y el método de rotación Varimax para (i) determinar las dimensiones subyacentes que emergen del conjunto de variables consideradas, (ii) determinar cuáles son los componentes más importantes y (iii), a la vez, identificar aquellas variables que podrían ser eliminadas de las dimensiones.

Para empezar, fueron chequeadas las condiciones de aplicación del ACP. La matriz de correlaciones apuntaba a la pertinencia de realizar el análisis factorial: algunas delas variables tenían correlaciones por encima de 0,3 (valor del coeficiente de correlación

10 Por las mismas razones, algunos pueden tener valores menores que 1 (pero siempre por encima de 0$)$. 
de Pearson). A la vez, no se daba multicolinealidad, es decir, correlaciones por encima del 0,9. Además, la prueba de esfericidad de Bartlett fue estadísticamente significativa (inferior a 0,05 ). En conjunto, estas pruebas demostraron la pertinencia de realizar un análisis factorial con el conjunto de variables.

El resultado del ACP fueron 6 dimensiones, pero se tomó la decisión de eliminar 2 de ellas, ya que no representaban restricciones a la libertad sino más bien trabas prácticas que encuentran e influencias que reciben los periodistas. En concreto, la primera de las dimensiones eliminadas se refería a limitaciones prácticas: tecnología, recursos, fechas tope y la otra, a influencias de parte de colegas, amigos, y otros. Dentro de las 4 dimensiones que sí respondían a restricciones, fueron eliminadas algunas de las variables, por criterios teóricos de consistencia y/o criterios estadísticos ${ }^{11}$.

Como resultado, aparecieron 4 dimensiones de restricción:

(1) La primera fue llamada "agentes externos" y contiene las siguientes variables: (i) oficiales del gobierno; (ii) políticos; (iii) hombres de negocios y (iv) líderes religiosos.

(2) La segunda dimensión se denominó "mercado y publicidad" y consta de: (i) consideraciones sobre la publicidad; (ii) expectativas de ganancias; (iii) el mercado y rating y (iv) auspiciadores.

(3) La tercera, "leyes y convenciones", contiene, tal como las dos anteriores, 4 variables: (i) convenciones de la sala de redacción; (ii) leyes de medios; (iii) convenciones profesionales y (iv) sindicatos de periodistas.

(4) La última dimensión, “agentes internos", consta de 3 variables: (i) superiores;

(ii) jefes y (iii) dueños.

11 De la primera dimensión fueron dejadas afuera dos variables, "censura" y "relaciones públicas", ya que todas las otras variables en esta dimensión, con excepción a estas dos, correspondían a actores concretos que coartan la libertad periodística, tales como políticos, representantes del gobierno, líderes religiosos y hombres de negocios. De esta forma, se especifica mejor la fuente de la restricción. "Relaciones públicas" tuvo, además, baja correlación con la dimensión, solo 0,405, según la matriz de componentes rotados, y si el valor es inferior a 0,440 se puede considerar la eliminación de la variable desde el punto de vista estadístico. Por no tener más de 4 variables en ninguna de las dimensiones, se eliminó de la dimensión 3 la variable cuya correlación con el componente fue la más baja: "sensibilidades". Las variables restantes fueron nuevamente sometidas a un ACP resultando, tal como se pensaba, en una división en 4 dimensiones. Sin embargo, nuevamente se procedió a eliminar una variable de la primera dimensión que esta vez tenía 5 variables, escogiendo la variable que tenía la correlación más baja con la dimensión: "observatorios de medios". 


\section{ANÁLISIS DE FRECUENCIA DE LOS ÍNDICES}

En esta parte, se dará a conocer la distribución de frecuencia del índice general y las cuatro dimensiones, para la totalidad de los países incluidos en la base de datos, para Chile y para los países democráticos incluidos en el estudio. Con fines comparativos, las frecuencias se presentan como gráficos.

GRÁFICO 1: Índice general de restricciones percibidas por los periodistas

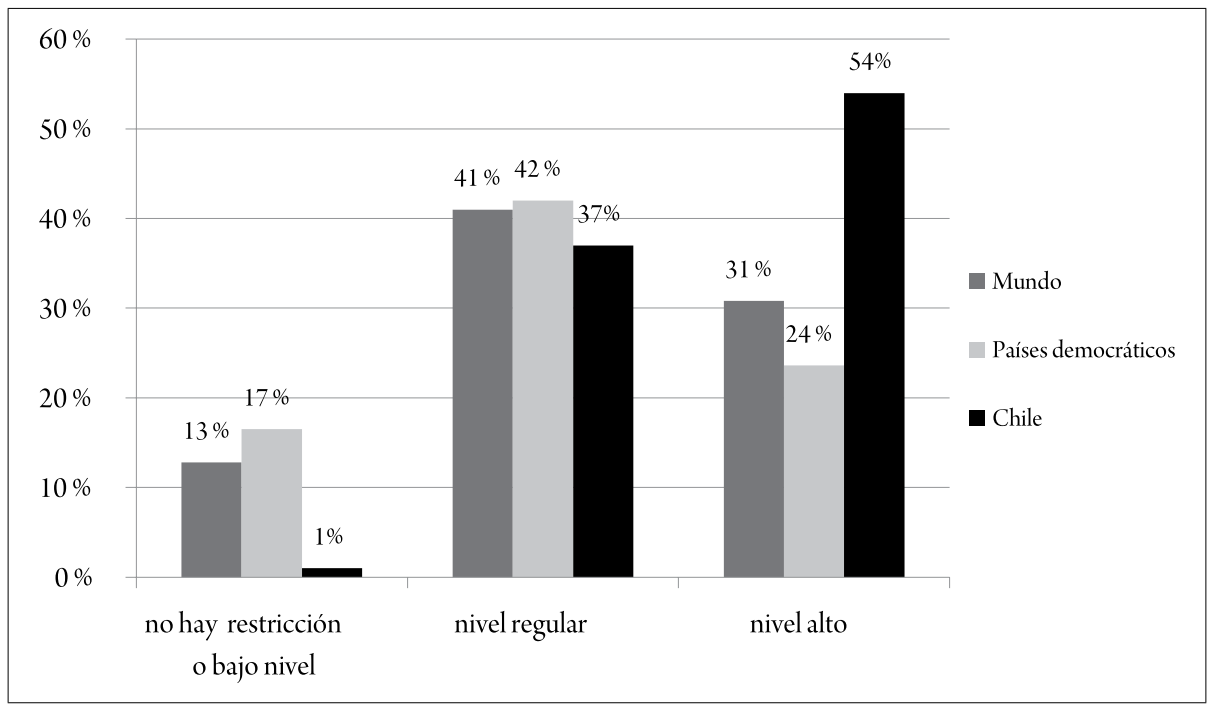

Elaboración propia sobre la base de datos recogidos por Worlds of Journalism (2012).

En el índice general vemos que mientras que en el mundo uno de cada tres periodistas percibe un alto nivel de restricción a su trabajo, en Chile lo hace más de la mitad. La diferencia entre Chile y los países democráticos es todavía más grande: en los países democráticos tan solo un $24 \%$ de los periodistas reporta niveles altos de restricción a su libertad, mientras que en Chile este porcentaje es más del doble: $54 \%$. Llama la atención además que tan sólo un uno por ciento de los periodistas chilenos se ubique en niveles bajos de restricción, comparado con un $17 \%$ en los países democráticos.

Se comprueba entonces la primera hipótesis que esperaba que entre un $40 \%$ y $50 \%$ de los periodistas a nivel global reportara niveles regulares de restricción, siendo este porcentaje un $41 \%$. No más de un tercio reporta niveles altos de restricción en el mundo, siendo este porcentaje un 31\%. En Chile, se esperaba que los niveles del índice general fueran altos para más de la mitad de los periodistas, y lo son para el $54 \%$. 
GRÁFICO 2: Dimensión de restricción por los agentes externos

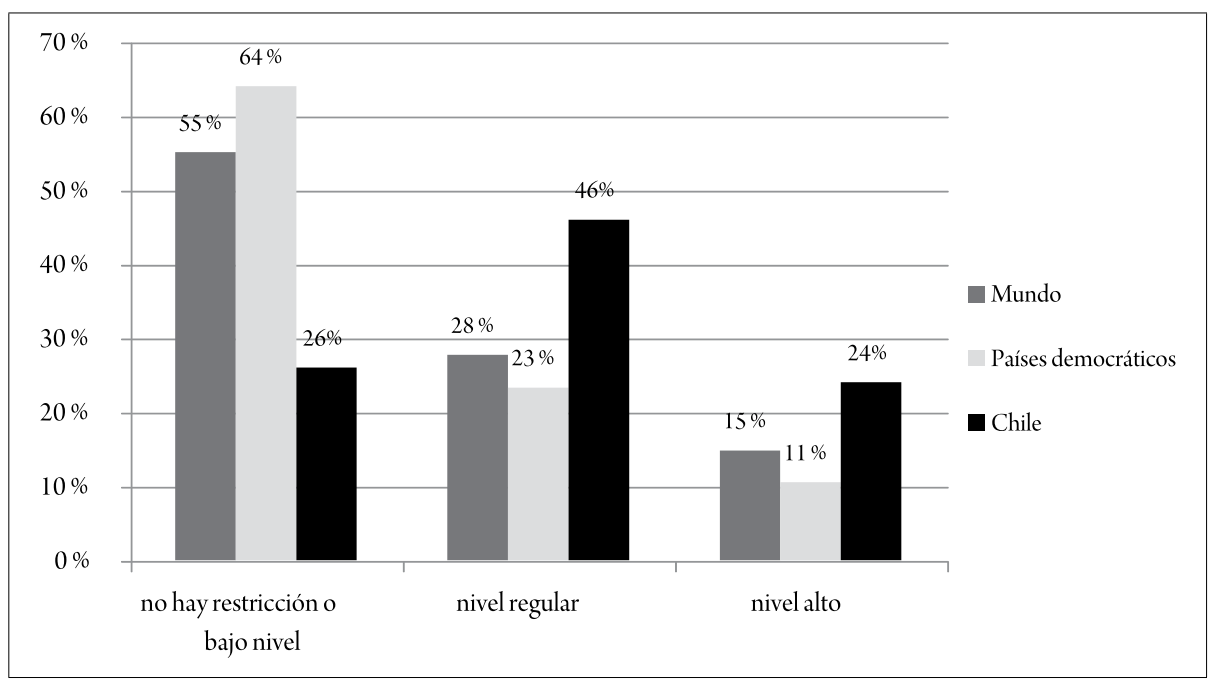

Elaboración propia sobre la base de datos recogidos por Worlds of Journalism (2012).

En esta dimensión, que se refiere a las limitaciones de parte de los políticos, hombres de negocios y líderes religiosos, vemos que la situación en Chile es nuevamente más preocupante que en el mundo en general y aún más si se compara solo con los países democráticos. Mientras que un 64\% de los periodistas en los países democráticos se ubica en el nivel bajo de restricción, lo hace tan solo un 26\% de los comunicadores chilenos. Y al contrario, el porcentaje que corresponde al nivel alto en Chile (24\%) supera más de dos veces al de los países democráticos (11\%).

\section{GRÁFICO 3: Dimensión de restricción por el mercado y la publicidad}

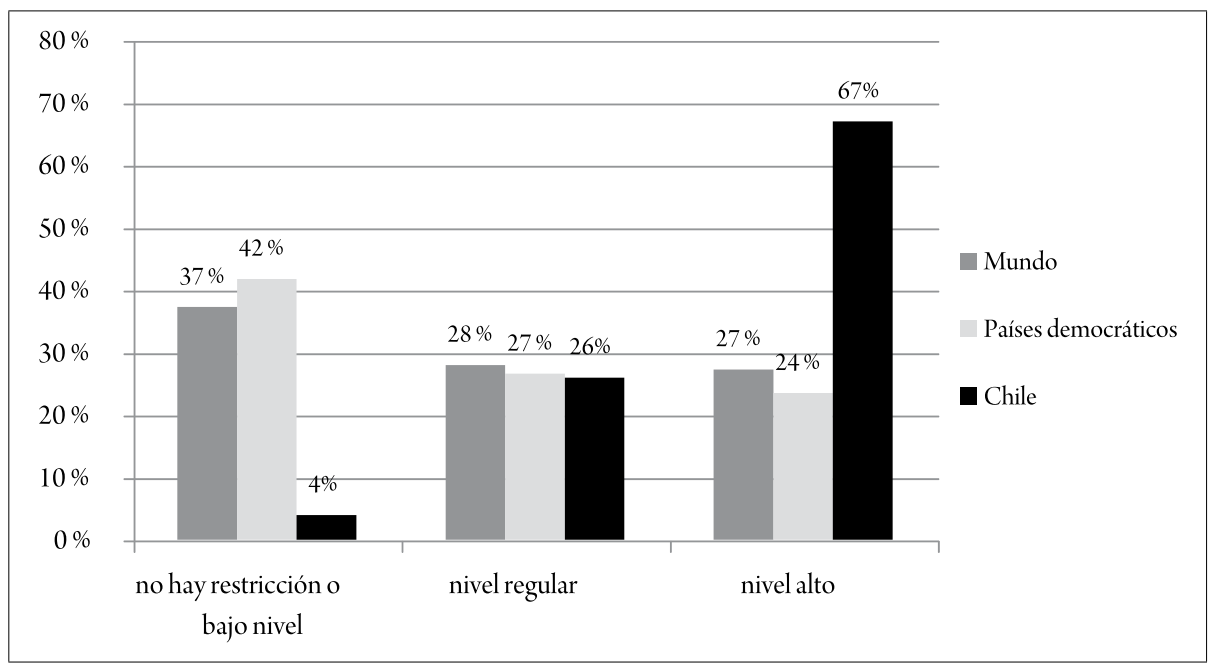

Elaboración propia sobre la base de datos recogidos por Worlds of Journalism (2012). 
En la dimensión de restricción por el mercado y la publicidad, las diferencias entre Chile y el mundo son todavía más marcadas. En el mundo un $27 \%$ de los periodistas se ubica en el nivel alto de restricción y en Chile más del doble de este porcentaje: un 67\%. Si comparamos Chile con los países democráticos, la diferencia es de 2,8 veces, cumpliéndose la hipótesis $\mathrm{H} 4$ que preveía que esta diferencia sería por lo menos el doble.

GRÁFICO 4: Dimensión de restricción por leyes y convenciones

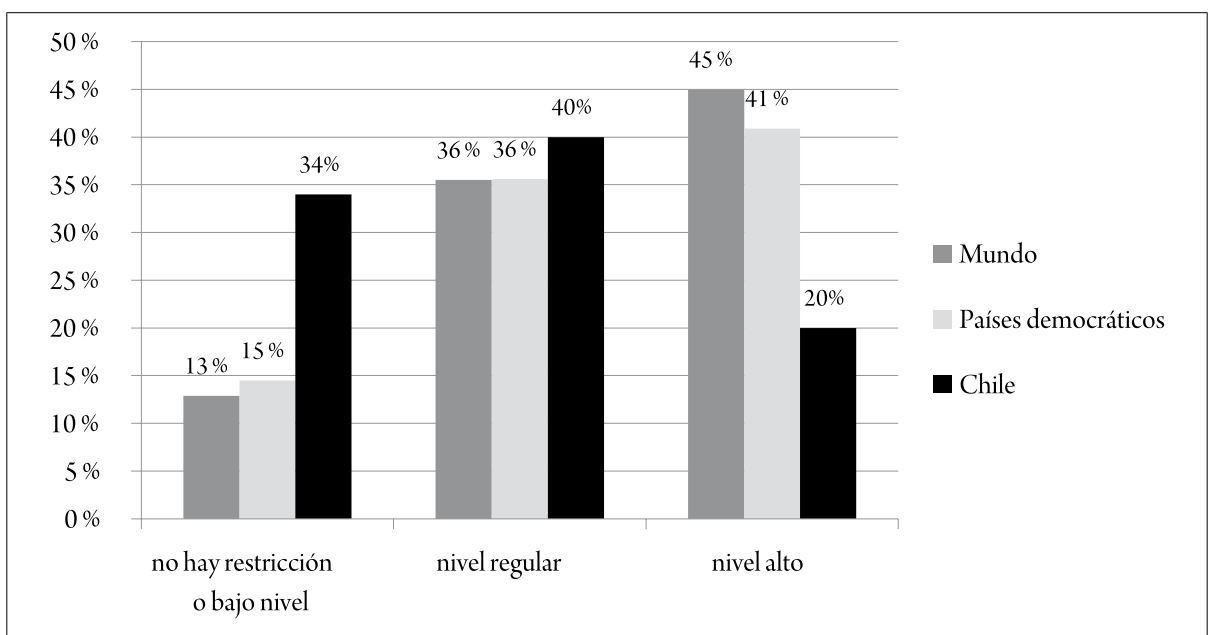

Elaboración propia sobre la base de datos recogidos por Worlds of Journalism (2012)

Sorprendentemente y abriendo preguntas para investigaciones futuras, los periodistas chilenos se ven menos restringidos por las leyes y convenciones no solo en comparación con el mundo, sino también con otros países democráticos.

GRÁFICO 5: Dimensión de restricción por agentes internos: superiores y dueños del medio

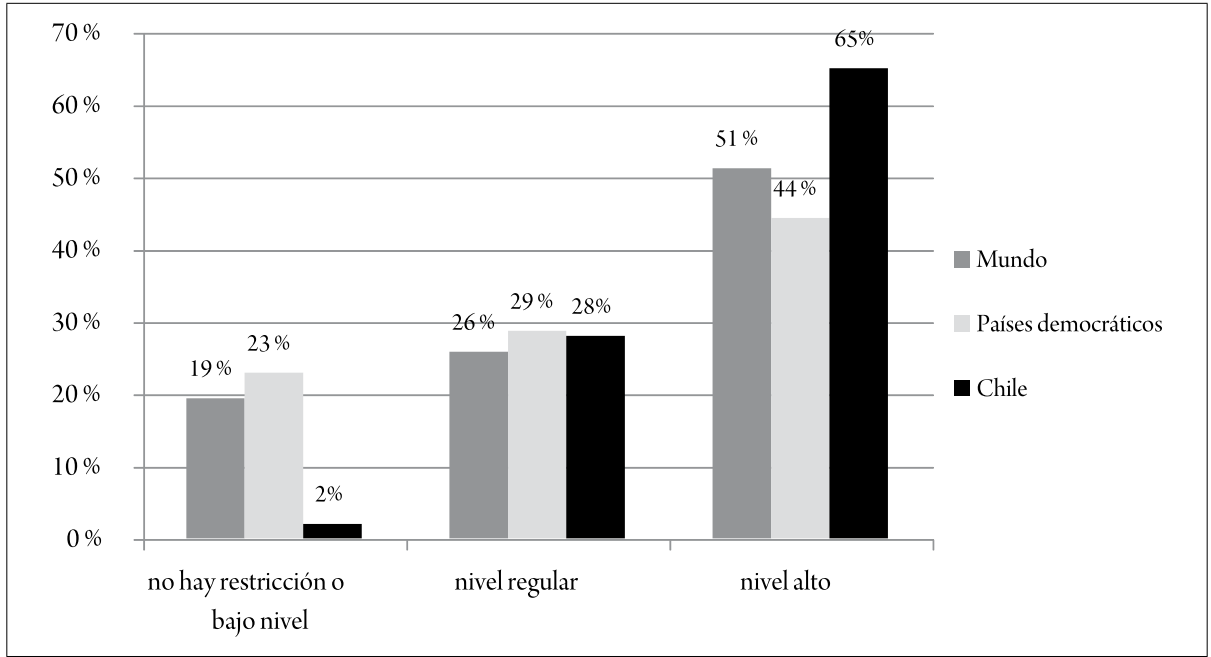

Elaboración propia sobre la base de datos recogidos por Worlds of Journalism (2012) 
Esta dimensión registra los más altos niveles de restricción percibida globalmente y en los países democráticos. Es llamativo que el porcentaje de los periodistas chilenos que se ubican en los niveles altos supere el porcentaje mundial y el de los países democráticos. De esta forma se comprueba la hipótesis $\mathrm{H} 2$ : en el mundo los niveles más altos de restricción se encuentran en el índice de restricciones por agentes internos (superiores y dueños del medio), llegando al nivel alto para más de la mitad de los periodistas (el 51\% exactamente). En Chile este índice tiene valores todavía más altos: un $65 \%$ de los periodistas reporta niveles altos de restricción.

Habiendo realizado la comparación de todas las distribuciones de frecuencia, se puede comprobar que la primera parte de la hipótesis H4: "Los niveles de restricción serán considerablemente más altos en Chile que en los países democráticos en general", se cumple para el índice general y para las dimensiones de agentes internos, externos y el mercado, y se rechaza para la dimensión de convenciones y leyes. Se cumple además la hipótesis H3: los niveles más altos en Chile se encontrarán en el índice de las restricciones por el mercado y la publicidad, ubicándose el $67 \%$ de los periodistas chilenos en el nivel alto en esta dimensión.

\section{PRINCIPALES RESULTADOS}

El resultado más importante del estudio fue comprobar que los niveles de restricción percibidos por los periodistas en Chile son altos y superan los mundiales, lo que abre preguntas profundas e inquietantes sobre el estado del periodismo en Chile y sus consecuencias democráticas. Por otro lado, demostrar que la dimensión dentro de la cual se registran mayores niveles de restricción en Chile es la del mercado y la publicidad urge a repensar la estructura del mercado mediático, el rol del Estado frente a él y la total dependencia de los medios de los ingresos del avisaje.

Se han comprobado todas las hipótesis, con la excepción de una parte de la hipótesis número 4 que preveía que los niveles de restricción en todas las dimensiones serían más altos en Chile que en otros países democráticos, en vista de que aquello no se cumple en la dimensión de leyes y convenciones. Sería interesante indagar en posteriores investigaciones a qué se debe el hecho de que los periodistas chilenos se vean menos restringidos por leyes y convenciones que sus colegas, a nivel global y los de otros países democráticos.

Se esperaba en la hipótesis $\mathrm{H} 1$ que entre un 40\% y un 50\% de los periodistas a nivel global reportase niveles regulares de restricción según el índice general y no más de un tercio reportase niveles altos, siendo los porcentajes recibidos 41 y 31 , respectivamente. En Chile se esperaba que los niveles del índice general fueran altos para más de la mitad de los periodistas y lo fueron efectivamente para un 54\%. Es decir, el nivel de las restricciones percibidas por los periodistas chilenos en su trabajo diario supera con creces los niveles globales. 
Tal como predijo la hipótesis $\mathrm{H} 2$, en el mundo los niveles más altos de restricción se encuentran en el índice de restricciones por agentes internos (superiores y dueños del medio), llegando al nivel alto para más de la mitad de los periodistas (exactamente para un 51\%). En Chile este índice tiene valores todavía más altos: un 65\% de los periodistas chilenos está dentro del nivel alto. Este es un resultado preocupante, sobre todo visto en el contexto de las precarias condiciones laborales de varios periodistas. ¿Cómo reaccionan los periodistas a estas presiones internas en vista de un mercado laboral altamente saturado y una desprotección sindical? Es una pregunta relevante para futuras investigaciones cualitativas.

A su vez, los niveles más altos en Chile, de acuerdo con la comprobada hipótesis $\mathrm{H} 3$, tiene la dimensión de las restricciones por el mercado y la publicidad, con un nivel alto para un $67 \%$ de los periodistas. Este porcentaje supera al global en 40 puntos porcentuales, apuntando a la estructura del mercado mediático y los modos de financiamiento de los medios en el país.

También en la dimensión de restricción de parte de los agentes externos políticos, hombres de negocios y líderes religiosos- los periodistas en Chile perciben más trabas que sus colegas en el mundo.

Además, tal como decía la hipótesis $\mathrm{H} 4$, los niveles de restricción son considerablemente más altos en Chile que en los países democráticos ${ }^{12}$, sobre todo en la dimensión referida al mercado y la publicidad. Mientras que en los países democráticos tan solo un $24 \%$ de los periodistas reporta niveles altos de restricción a su libertad según el índice general, en Chile este porcentaje es más del doble: $54 \%$, lo que contradice los buenos resultados que obtiene Chile en los índices de libertad de prensa de RSF y Freedom House.

La dimensión de restricción por el mercado y la publicidad, a la que hace referencia la hipótesis, tiene nivel alto para el 24\% de los periodistas en los países democráticos y para el $67 \%$ de los periodistas chilenos, superando casi 3 veces el resultado de los países democráticos. Esto viene a confirmar las conclusiones de Hanitzsch y Mellado (2011) y demuestra la importancia de incluir esta dimensión en las mediciones de la libertad de prensa y la libertad de expresión. También la dimensión de restricción por agentes internos (jefes y dueños) tiene valores considerablemente más altos en Chile, que en los países democráticos: un 65\% de los periodistas dentro del nivel alto de restricción frente a un 44,3\%. Lo mismo ocurre en la dimensión de restricción por parte de los agentes externos, donde un $24 \%$ de los periodistas chilenos se ubica en niveles altos: 2,4 veces más que en los países democráticos.

12 Con la ya mencionada excepción de la dimensión de restricción por leyes y convenciones. 


\section{CONCLUSIONES}

Resumiendo los alcances del presente estudio, se ha logrado elaborar índices del nivel de restricción que perciben los periodistas en el mundo, en los países democráticos y en Chile. Luego, las distribuciones de frecuencia posibilitaron comparar Chile con el mundo y con otros países democráticos. De esta forma, se contribuyó a reforzar los escasos análisis comparativos de este tipo y se hizo una propuesta metodológica frente a la escasez de índices académicos de restricciones a la libertad de expresión y prensa.

El estudio propuso además, a base de un análisis factorial y las posteriores decisiones con criterio teórico y estadístico, cuatro dimensiones de restricciones de la libertad periodística: por (i) agentes externos; (ii) por el mercado y la publicidad; (iii) por leyes y convenciones y (iv) por agentes internos. Si tuviéramos que establecer una jerarquía de estas dimensiones basada en su peso relativo (calculado como porcentaje de casos en el nivel alto de restricción) para el mundo en general y también para los países democráticos las dimensiones en orden priorizado serían: agentes internos, leyes y convenciones, mercado y publicidad, y finalmente, agentes externos; lo que daría la razón a los que insisten en la importancia primordial del ámbito organizacional (Tuchman, 1978). Sin embargo, para Chile este orden sería distinto: el mercado y la publicidad en primer lugar, luego agentes internos, agentes externos $y$, al final, leyes y convenciones; lo que confirmaría los escritos de los economistas políticos de la comunicación (Murdock, 2001).

El estudio ha demostrado que los niveles de restricción de la libertad periodística son considerablemente más altos en Chile que en el mundo y la diferencia aumenta si se compara Chile únicamente con otros países democráticos. Además, en ciertas dimensiones, como la del mercado, el nivel de restricción en Chile es varias veces mayor al de los países democráticos. Es un resultado relevante para el debate sobre el estado del periodismo, las restricciones a la libertad de expresión en los medios de comunicación en Chile, la estructura del mercado mediático y, en fin, la calidad de la democracia.

Los altos niveles de restricción percibidos por más de dos tercios de los periodistas chilenos de parte del mercado y la publicidad, hacen que la misión del periodismo de informar sobre asuntos públicos de importancia pierda frente al objetivo de vender, de atraer -y mantener- la publicidad. También los altos niveles de restricción por parte de los superiores y dueños del medio, conjugados con la saturación del mercado laboral para periodistas, la concentración económica de los medios en Chile y-en en el caso de la prensa escrita-su uniformidad ideológica (Baltra, 2012), tienen claras consecuencias negativas para el derecho a la información de la ciudadanía y para el debate público. 


\section{Referencias bibliográficas}

AMARC. (2009). Las mordazas invisibles. Nuevas y viejas barreras a la diversidad de radiodifusión. Buenos Aires: AMARC.

Baltra, L. (2012). La prensa chilena en la encrucijada. Entre la voz monocorde y la revolución digital. Santiago: LOM.

Blumler,J.y Gurevitch, M. (1990). Political Communication Systems and Democratic Values. En Lichtenberg, J. (Ed.), Democracy and the Mass Media. Cambridge: Cambridge University Press.

Breed, W. (1955). Social Control in the Newsroom: a Functional Analysis. Social Forces, 33(4), 326-335.

Cabalin, C. y Lagos, C. (2009). Libertad de expresión y periodismo en Chile: presiones y mordazas. Palabra Clave, 12(1), 37-59.

Corrales Jorquera, O. y Sandoval Moya, J. (2005). Concentración del mercado de los medios, pluralismo y libertad de expresión. Santiago: Fundación Chile 21.

Cottle, S. (Ed.). (2003). News, Public Relations and Power. Londres: Sage Publications.

Cottle, S. (2008). Reporting demonstrations: the changing media politics of dissent. Media, Culture \& Society, 30(6), 853-872.

Freedom House (2011). Freedom of the Press 2011. www.freedomhouse.org/ report/freedom-press/2011/chile

Gumucio, M. y Parrini, V. (2009). Política comunicacional de la Concertación. En Quiroga, Y. y Ensignia, J. (Eds.), Chile en la Concertación 1990-2010. Una mirada crítica, balance y perspectivas (pp. 301-331). Santiago: FriedrichEbert-Schtiftung.

Hanitzsch, T. y Mellado, C. (2011). What Shapes the News around the World? How Journalists in Eighteen Countries Perceive Influences on Their Work. The International Journal of Press/Politics. Obtenido 28 de junio de 2013 en http://hij.sagepub.com/content/early/2011/04/28/1940161211407334

Hanitzsch, T. et al. (2010). Modelling Perceived Influences on Journalism: Evidence from a Cross-National Survey of Journalists. Jornalism and mass Communication Quarterly, 87(1), 5-22.

King, G. et al. (2012). How Censorship in China Allows Government Criticism but Silences Collective Expression. Working Paper. Obtenido 28 de junio de 2013 en http://j.mp/LdVXqN

Lagos, C.y Cabalín, C. (2009). Condiciones profesionales del ejercicio del periodismo y de la libertad de expresión en los principales medios de comunicación de la Región Metropolitana. Santiago: ICEI, Universidad de Chile.

Lagos, C., Matus, A. y Vera, M. (2005). Organizaciones Sociales en Chile invisibles a los medios. Santiago: ICEI, Universidad de Chile.

Latinobarómetro. Análisis en línea. www.latinobarometro.org/latino/LATAnalize.jsp 
Mellado, C., del Valle, C., Salinas, P., \& González, G. (2009). Encuesta de Periodistas de Prensa. Medios de Comunicación. Santiago: USACH y Otros.

Mellado, C. (2011). The Chilean Journalist. En Weaver, D. \& Wilnat, L. (Eds.), The Global Journalsit in the 21st Century: News People Around the World (pp. 382-412). Nueva York: Routledge.

Murdock, G. (1999). Rights and Representations: Public Discourse and Cultural Citizenship En Gripsrud, J. (Ed), Television and Common Knowledge (pp. 7-17). London: Routledge.

Murdock, G. (2001). Political Economy of Mass Media. En Smelser, N. y Baltes, P. (Eds.), International Encyclopedia of the Social and Behavioural Sciences (pp. 9358-9363). Oxford: Elsevier Science

Otano, R. y Sunkel, G. (2003). Libertad de los periodistas en los medios. Comunicación y medios, 14.

Rebolledo, L. (2000). Percepciones de los sectores populares sobre la libertad de expresión. Santiago: ICEI, Universidad de Chile.

RSF (2010). Clasificación Mundial 2010. Obtenido 28 de junio de 2013 en http: / / es.rsf.org/press-freedom-index-2010,1034.html

RSF (2012). Clasificación Mundial 2011-12. Obtenido 28 de junio de 2013 en http:/ / en.rsf.org/press-freedomindex-2011-2012,1043.html.

Salinas, C., Stange, H. y Faure, A. (2011). El imperio del sentido común: influencia del sistema mediático sobre las prácticas profesionales de los periodistas 1970-2000. Confederación Iberoamericana de Asociaciones Científicas y Académicas de la Comunicación CONFIBERCOM.

Sapiezynska, E. (2011). Lucha por la palabra en América Latina. Revista noruega $X$-Verdensmagasinet, 3, 54-56.

Sapiezynska, E. (2012). La libertad de expresión y los medios de comunicación en Chile hoy: desafíos actuales para los estudios de la comunicación política. Santiago: ACCP.

Sapiezynska, E., Lagos, C. y Cabalín, C. (2013). Libertad de prensa bajo presión: niveles de restricción percibidos por periodistas chilenos y factores influyentes. Cuadernos de Información, 32, 11-26.

Schudson, M. (2002). The Media as Political Institutions. Annual Review of Political Science, 5, 249-264.

Shoemaker, P. y Reese, S. (1996). Mediating the message: Theories of influence on mass media content. New York: Longman.

Tuchman, G. (1978). Making News. Nueva York: The Free Press.

UDP (2008). Informe Anual sobre Derechos Humanos en Chile 2008. Santiago: UDP.

UDP (2010). Estudio Nacional sobre Lectoría de medios escritos. Santiago: UDP.

UDP (2012). Encuesta Nacional. Santiago: UDP.

Worlds of Journalism (2012). www.worldsofjournalism.org/ 